\title{
Muundo WA Virai Vya Sentensi Ya Kî̀gembe
}

\author{
Mutwiri Fred John M. A ${ }^{1}$, Allan Mugambi, Ph.D ${ }^{2^{*}}$ \\ ${ }^{1}$ Department of Humanities, Faculty of Humanities and Social Sciences, Chuka University, Kenya \\ ${ }^{2}$ Department of Humanities, Faculty of Humanities and Social Sciences, Chuka University, Kenya
}

Article History

Received: 21.01.2021

Accepted: 04.03.2021

Published: 23.03.2021

Journal homepage:

https://www.easpublisher.com

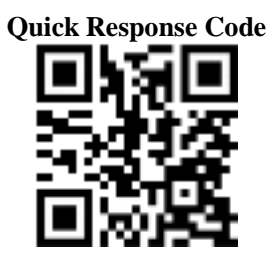

Abstract: Kirai ni kipashio cha muundo chenye neno moja au zaidi lakini kisicho na muundo wa kikundi nomino na kikundi tenzi. Virai ni mojawapo ya vipashio vya sentensi ya Kî̀gembe vinavyoshughulikiwa katika taaluma ya sintaksia. Makala hii inashughulikia muundo wa virai vya sentensi ya Kî̀gembe kwa kufafanua kanuni zinazoongoza uunganishwaji wa virai kuundia sentensi ya Kî̀gembe.Data ya sentensi 50 imetumika katika makala hii. Virai aina tano vilichanganuliwa na kuainisha miundo ya virai katika Kî̀gembe na kuwasilishwa kwa njia ya maelezo. Makala hii inachangia katika kuongeza maarifa ya kiutafiti kuhusu sentensi ya Kî̀gembe, kutumika kwa lugha hii kwa kuchangia maendeleo endelevu na kuhifadhi maandishi yake.

Keywords: Eksibaa, Kî̀gembe, virai, vipashio.

Copyright (C) 2021 The Author(s): This is an open-access article distributed under the terms of the Creative Commons Attribution 4.0 International License (CC BY-NC 4.0) which permits unrestricted use, distribution, and reproduction in any medium for non-commercial use provided the original author and source are credited.

\section{UTANGULIZI}

Mkude [2], anaeleza virai kama viambajengo vinavyounda sentensi mbalimbali. Anarejelea virai kama vile virai nomino, virai vivumishi, virai vihusishi, virai tenzi na virai vielezi. Virai hivi huunda vishazi mbalimbali kama vile vishazi tegemezi, vishazi ambatano na vishazi huru ambavyo huunda sentensi Kî̀gembe. Kwa mujibu wa Besha [3], maarifa aliyonayo mjua lugha ni pamoja na kujua muundo sahihi wa sentensi za lugha yake. Sentensi inafafanuliwa kama tungo iliyo kamili kisarufi, isiyotegemea mazingira ya mawasiliano ili kueleweka kikamilifu. Sifa kuu za uchambuzi wa sentensi ni kubaini mfuatano wa maneno katika sentensi, uainishaji wa maneno katika makundi, kubaini uhusiano wa maneno yanayojenga sentensi, na kubaini uhusiano kati ya sentensi. Sentensi inaundwa na sehemu kuu mbili mathala: kundi nomino na kundi tenzi. Muundo wa sentensi hujitokeza kama:

Sentensi (S) - Kundi Nomino $(\mathrm{KN})+$ Kundi Tenzi (KT). Hivyo basi kirai ni kiungo muhimu katika uundaji wa sentensi kwani ni mojawapo wa viambajengo vinavyounda sentensi.

\section{Miundo ya Virai vya Lugha za Kibantu}

Lugha za kibantu huwa na ukuruba katika miundo na hata maana. Kiigembe ni mojawapo wa lugha za kibantu. Lugha zingine za kibantu ni pamoja na Kigikuyu, Kimeru, Ekegusii, Kiswahili miongoni mwa zingine. Wataalamu wengu wamefanya ufatiti mwingi kuhusu muundo wa lugha ya Kiswahili hata hivyo. Besha [3] alibainisha vipashio vikuu vijengavyo sentensi kwa kushughulikia virai kama vile virai nomino, virai vivumishi, virai vitenzi na virai vihusishi. Anasema kuwa vipashio hivi vikihusishwa na muundo wa sentensi hujitokeza katika muundo wa sentensi sahili, sentensi ambatano ambayo huwa ni sentensi sahili mbili na sentensi changamano. Massamba na wenzake [4], waliainisha virai vya Kiswahili kama vile, kirai kivumishi, kirai kielezi, kirai nomino na kirai kihusishi. Japo walijihusisha na kanuni miundo virai iliyo katika Nadharia ya Sarufi Zalishi Geuza Maumbo (SZGM). Aidha, wanasema kuwa, sentensi ni kipashio kikubwa kabisa cha kimuundo ambacho kimekamilika kimaana. Mpangilio wa maneno katika sentensi unafanywa kwa utaratibu maalum kufuatwa na neno lingine ambalo lina uhusiano nalo. Miundo ya sentensi huhusisha vipashio vingine vidogo kimuundo. Vipashio hivyo ni viambajengo vya sentensi kama vile; virai. Wanasema kwamba muundo wa msingi wa sentensi huwa unahusisha virai tu, na hasa kirai Nomino na kirai Kitenzi. 
Masamba na wenzake [4], wamejadili virai katika Kiswahili sanifu kwa kuonyesha kwamba kirai huundwa kutokana na mahusiano maalumu baina ya maneno. Mahusiano hayo huwa ni ya asili katika lugha na hujidhihirisha kisarufi. Muundo wa kirai hukitwa kwa neno kuu katika lugha kama vile nomino, vitenzi, vivumishi, vielezi na hata viunganishi. Maneno haya makuu ndiyo hutupatia aina mbalimbali za virai. Kiswahili kina virai kama vile: virai nomino, virai vitenzi, virai vielezi, virai vivumishi, virai viunganishi $[5]$.

Njogu, Mwihaki na Buliba [6], wanasema kuwa, miundo ya sentensi huhusisha vipashio vidogo kimiundo. Vipashio hivyo ni neno, virai na vishazi. Muundo wa msingi wa sentensi huhusisha virai tu, na hasa kirai nomino na kirai tenzi. Pia, Kirai Kivumishi, Kirai Kielezi na kirai kihusishi. Kuna miundo ya sentensi inayohusisha miundo tofauti ya msingi iliyounganishwa kwa njia mbalimbali. Makala hii ilibainisha muundo na mpangilio wa viambajengo vya sentensi ya Kiigembe kama vile, neno, kirai na kishazi. Darajia hizo ni sauti ambazo husukwa kwa kanuni ya lugha husika na kuunda maneno. Maneno pia kwa utaratibu wa lugha husika yana kanuni ambayo hufuatwa katika kuyaunda na kuyafuatisha ili yaunde virai. Virai huunda vishazi ambavyo huunda sentensi [7].

Kuonyesha muundo wa kirai kitenzi katika lugha huonyesha ni vipashio gani vinaandamana na kitenzi katika kuunda tungo. Kî̀gembe huwa na kirai tenzi ambacho ni kiambajengo cha sentensi ya Kî̀gembe. Kirai cha Kî̀gembe hutegemea neno kuu yaani kichwa katika kirai husika kama ilivyofafanuliwa kwa kuegemea mwongozo wa Nadharia ya Eksibaa kwa mujibu wa Chomsky [1]. Radford [8], anasema kuwa, kila mahali ambapo kirai kinatokea lazima kuwe na kategoria ya leksia. Kategoria hizi za maneno ndizo hubeba sifa za kirai. Hii ni kumaanisha kuwa, kila kunapotea kirai nomino lazima kuwe na nomino

\section{Misingi ya Kinadharia}

Nadharia ambayo ilitumika katika makala hii ni Nadharia ya Eksibaa. Nadharia ya Eksibaa iliasisiwa na Chomsky [1]. Nadharia hii inajikita katika mfumo wa muundo virai. Nadharia hii iliibuka ili kueleza kirai kwa ukamilifu baada ya Sarufi Miundo Virai kuonekana kuwa na upungufu wa kuwa na kanuni nyingi. Aidha, Nadhari ya Eksibaa huongozwa na dhana ya kichwa (neno kuu) katika kirai na uhusiano wake na vipengele vingine ambavyo vimetumika kuunda kirai. Nadharia ya Eksibaa husaidia kuchanganua sentensi kwa kutekeleza majukumu kama vile; kubainisha muundo wa kirai, kuelezea mahusiano ya vipashio katika kirai, kuonyesha jinsi umilikaji unavyojitokeza baina ya vipashio na kuonyesha matumizi ya kanuni za kijumla katika kufafanua muundo wa kirai.
Kwa mujibu wa Chomsky [9], Nadharia ya Eksibaa hutumika katika uchanganuzi wa sentensi ili kuwezesha ujumlishaji wa sifa zinazojitokeza katika aina mbalimbali za virai kama vile virai nomino, virai vitenzi, virai vivumishi, virai vielezi na virai vihusishi. Alama ya (X) husimamia kichwa au neno kuu katika kirai na alama ya ritifaa (') (baa) husimamia vipashio vinavyofuatana na neno kuu (X') katika kirai husika.

Kulingana na Chomsky (keshatajwa), muundo wa virai huchukuliwa kuwa na viwango vitatu vya uchomozi ambavyo ni; kiwango cha uchomozi wa neno, kiwango cha uchomozi wa kati na uchomozi wa upeo. Kiwango cha uchomozi upeo huwakilishwa na herufi ya neno kuu katika kirai pamoja na baa mbili juu yake $(X ")$. Kwa upande mwingine uchomozi wa kati huwakilishwa na herufi ya neno kuu katika kirai pamoja na baa moja (X'). Uchomozi wa neno huwakilishwa na alama (X).

Kwa mujibu wa Cowper [10], Nadharia ya Eksibaa ni nadharia ya Sarufi Miundo iliyoendelezwa badala ya ile ya Sarufi Miundo Virai. Hoja muhimu katika nadharia hii ni kwamba sheria za Sarufi Miundo Virai hazina budi kudhibitiwa; na kwamba kategoria zaidi za virai hazina budi kuongezwa. Udhaifu wa kanuni miundo virai ni kuwa kanuni hazikudhibitiwa na kwamba zilichukua muundo huru. Kauli ya Cowper (keshatajwa) inalingana na maoni ya Newmeyer [11], anayesema kuwa, Nadharia Eksibaa ilitokana na ile hali ya kupata suluhisho baada ya kufahamika kuwa Nadharia ya Sarufi Miundo Virai ilikuwa na kanuni nyingi zilizohitaji kudhibitiwa.

Nadharia ya Eksibaa inaonyesha kwamba, katika lugha zote, kila kirai huwa na kichwa lakini mpangilio wa vipashio vinavyofuatana na kichwa hicho hutegemea lugha maalum. Kwa mfano; 'mtoto wangu,' kichwa ni nomino 'mtoto' ilhali katika kirai kitenzi 'ameandika vizuri,' kichwa ni kitenzi 'ameandika.' Kwa hivyo, kanuni kuu ya nadharia hii ni: KX ...X... ambayo inaonyesha kwamba kila kirai KX lazima kiwe na kichwa cha $X$ ili kirai chenyewe kiwe sahihi. Kwa hivyo KX ...N.. na KX ...T...ambapo $\mathrm{N}$ ni nomino na $\mathrm{T}$ ni kitenzi.

Kulingana na Radford [12], kuna viwango vitatu vya kuchanganulia virai Kategoria ya kiwango cha neno, kategoria ya kiwango cha kati na kategoria ya kiwango cha kirai. Aidha, anabaini kuwa kategoria za kati huwa kubwa kuliko kategoria kileksia lakini ndogo kuliko Kategoria kamili (kirai kikuu). Kuna viambajengo vilivyo vikubwa kuliko nomino lakini vidogo kuliko kirai nomino.

Kirai nomino lazima kiwe na nomino, kirai tenzi lazima kiwe na kitenzi, kirai husishi lazima kiwe na kihusishi, kirai elezi lazima kiwe na kielezi na kirai vumishi lazima kiwe na kivumishi. Kipashio ambacho 
ni cha lazima katika kikundi fulani cha kirai kinaitwa (kichwa) cha kategoria. Sifa za kichwa zinakuwa sifa za kirai chote. Kichwa huamua upatanisho wa kisarufi katika kirai.

Kwa kuzingatia RN, RT, KV, KE na KH inawezekana kuwa na kirai ambacho kina kichwa pekee [10] virai hivi vyote, kipashio muhimu na cha lazima ni kichwa cha kirai. Vipashio vingine vyote ni huria (si vya lazima). Nadharia ya Eksibaa inaongozwa na dhana ya neno kuu (kichwa) katika kirai na uhusiano wake na vipengele vingine katika kuunda kirai. Katika Nadharia ya Eksibaa, alama $X$ hutumika kuwakilisha neno kuu katika kirai na (') au baa huwakilisha vipashio vifuatilizo vya neno kuu/kichwa katika kirai husika. Na $\mathrm{X}$ ' huwakilisha kirai cha $\mathrm{X}$. Maelezo haya yanaweza kuelezwa kwa mchoro ufuatao,

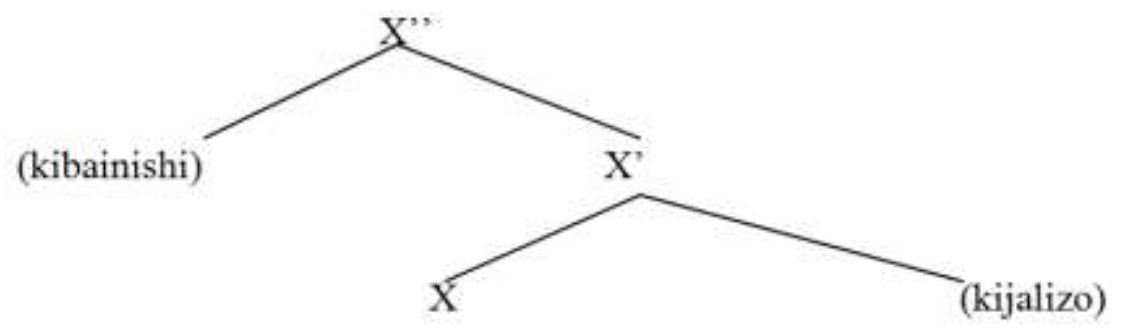

$\mathrm{X}$ ni kategoria ya maneno kama vile $\mathrm{N}$ (nomino), $\mathrm{T}$ (kitenzi), $\mathrm{H}$ (kihusishi), $\mathrm{V}$ (kivumishi), $\mathrm{E}$ (kielezi). Kibainishi na kijalizo si za kategoria leksia bali huwakilisha mahusiano. Kibainishi ni neno linalotumika pamoja na nomino [13]. Kulingana Radford [12], anasema kuwa, Jukumu la kibainishi katika nadharia ya Eksibaa ni kupanua $\mathrm{N}$ ' hadi $\mathrm{N}$ ', katika kielelezo tungo. Mabano yaliyowekwa kwenye kibainishi na kijalizo yanadhihirisha kuwa vipengele hivi ni huria na wala si vya lazima.

Nadharia ya Eksibaa inazingatia upangaji wa vipashio mbalimbali vya kileksia katika viwango tofauti katika sentensi. Ni upangaji unaobaini ni kipashio kipi kina uhusiano wa ngazi moja na ni kipi kinamiliki kingine. Radford [12], alitumia istilahi kama vile;

a) dada (viambajengo vilivyo katika ngazi moja) b) binti ya (viambajengo vinavyomilikiwa na vingine)

c) shangazi (uhusiano usiotokana na kifundo kimoja)

d) kifundo (uhusiano katika kiwango cha udada)

e) kijalizo (kipashio kinachofafanua kichwa cha kirai katika kirai husika)

f) kibainishi (kategoria ya kileksia na hasa hutumika na nomino)

g) spesifaya (neno linalotumika na nomino na kwa kurejelea kategoria leksia huitwa kibainishi)

h) chagizo (sehemu inayoonyesha uhusiano wa uelezi)

Istilahi hizi zinaweza kuelezwa vyema kwa kutumia mchoro ufuatao;

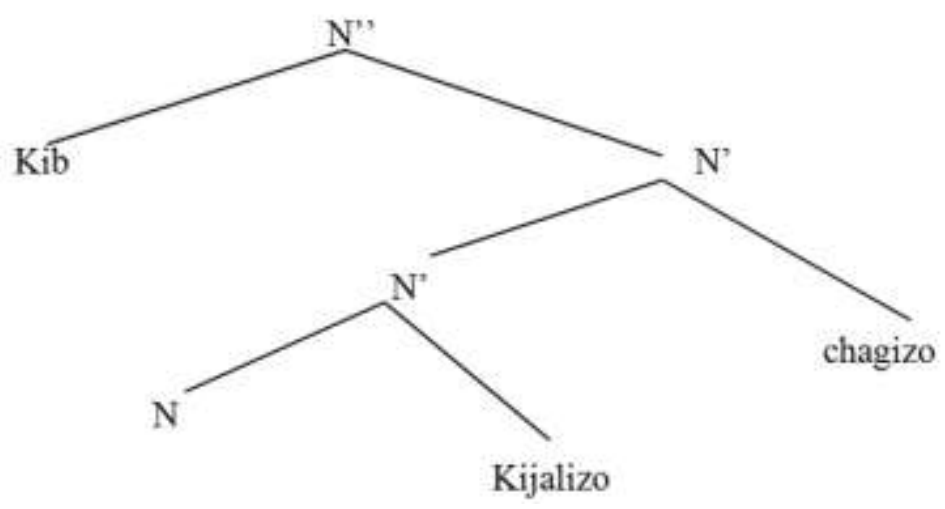

Katika mchoro huu, (kibainishi) ni dada ya N' na dada hao wawili yaani kibainishi na N' ni binti za $\mathrm{N}$ ', Wanamilikiwa na $\mathrm{N}$ '. Chagizo ni dada na binti ya $\mathrm{N}$ ' na kijalizo ni dada ya $\mathrm{N}$ na binti za $\mathrm{N}$ ' chagizo ni shangazi ya N. Kijalizo hupanua N hadi N'. Nacho chagizo hupanua N' hadi N'. Kibainishi hupanua N' hadi $\mathrm{N}$ '. Kutokana na maelezo haya inadhihirika kuwa uchanganuzi wa Eksibaa unatusaidia kueleza uhusiano wa viambajengo kwa kuzingatia muundo pekee. Ufafanuzi zaidi unadhihirika katika kielelezo kifuatacho;

S1 Ûrîa mwini wa rwîmbo kuuma Mutuati. (Kị̂̂embe ) Yule mwimbaji wa wimbo kutoka Mutuati. (Kiswahili) 


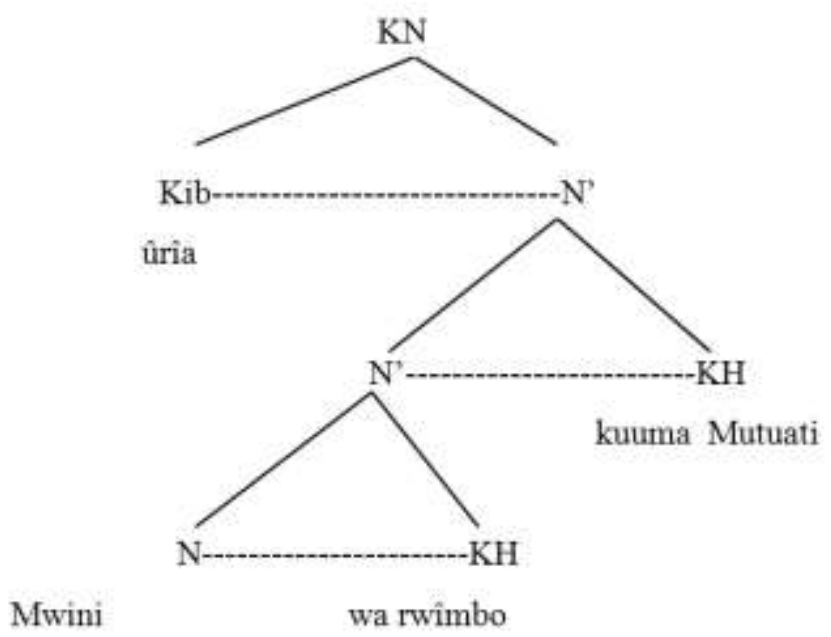

Kipashio KN kinamiliki vifundo vya kibainishi na N'. Kifundo cha kibainishi na cha N' ni cha kiwango kimoja (dada). N KH (Kijalizo) ni vipashio vya ngazi moja na vinamilikiwa na N'. N' na KH (chagizo) pia ni vipashio vya ngazi moja na vinamilikiwa na N'. Kwa kuzingatia maelezo ya mchoro huu uhusiano baina ya nomino $\mathrm{N}$ na chagizo ni wa mbali. Lakini kuna uhusiano wa karibu sana kati ya kichwa $\mathrm{N}$ na kijalizo, hii ndiyo sababu haiwezekani kukitenganisha kijalizo na kichwa cha kirai na kukihamisha hadi mwisho wa kirai.

Kwa mujibu wa Leffel na Bouchard (1991), Nadharia ya Eksibaa huwakilishwa na neno kuu ambalo linaweza kuwa, kitenzi, nomino, kielezi, kivumishi na kihusishi kwani ndio hubeba sifa za kirai. Kwa hivyo, kirai kitakuwa na sifa za kategoria fulani ya neno ambalo ndilo neno kuu la kirai hicho. Kwa mfano, kirai nomino (RN) kiini chake kitakuwa ni nomino (N) ambayo itachukuwa nafasi ya $\mathrm{X}$ katika muundo wa kirai kilivyotumika katika utafiti huu. Kwa mfano;

S2 Mwarimû nîakûritana - Kî̀gembe

Mwalimu anafundisha - Kiswahili

Katika mfano huu, neno mwarîmû ni nomino ambayo imesimama kama neno kuu la kirai nomino na wakati huo huo imesimama kama kirai nomino katika fungu hilo. Nadharia ya Eksibaa inaeleza kuwa, muundo wa kirai unaweza kuwa na mchomozo mpana wa kibainishi kimoja au zaidi. Hali hii hujitokeza kwa neno kuu kama nomino kufuatwa na kibainishi kingine katika muundo wa kirai. Mfano ufuatao kutoka Kî̀gembe unafafanua dhana hii:

S3 Ngûkûû îrîa îrareterwe îoro îukua- Kiigembe

Kuku aliyeletwa jana amekufa- Kiswahili

Mfano huu unaonyesha kuwa sentensi ya Kiigembe inaweza kuwa na mchomozo mpana kwa kuchukua vibainishi zaidi. Kwa kuangalia umuhimu wa kuchanganua sentensi na virai kwa kutumia nadharia inayoangalia kategoria kama hii ya Eksibaa, iliweza kuchanganua miundo ya vishazi na virai mbalimbali vya sentensi ya Kî̀gembe. Hii ni kwa sababu, sentensi huundwa kwa mpangilio maalumu ambao huwa na virai ambavyo vimewekwa katika seti maalumu ya kategoria. Virai na vishazi ni sehemu muhimu ya sentensi ya Kî̀gembe.

Mihimili ya Nadharia ya Eksibaa

Mihimili ifuatayo iliweza kutumika katika utafiti wa kazi hii. Hii ni pamoja na;

(i) Kila kirai huwa na neno kuu

Kulingana na Leffel na Bouchard [14], nadharia ya Eksibaa huwakilishwa na neno kuu katika kirai ambalo hubeba sifa za kirai kile. Neno kuu katika kirai nomino cha Kî̂gembe huwa ni neno la kwanza katika kirai nomino, neno kuu ni nomino au kiwakilishi cha nomino. Kwa mfano;

S4 Mwaana ûmûkuî aûkûlûka - Kiigembe

Mtoto mfupi amepita - Kiswahili

Neno kuu la kirai nomino ni "mwaana"

Radford [12], anasema kuwa, kila mahali kunapotokea kategoria ya kirai ni lazima kuwe na kategoria ya leksia. Hii inamaanisha kila mahali ambapo kwa mfano; kirai nomino kinapotokea lazima kuwe na nomino na kila kirai kihisishi kinapojitokeza lazima kiwe na kihisishi na kila kirai tenzi kinapojitokeza lazima kuwe na kitenzi. Vipashio hivi yaani, nomino, kitenzi, kihisishi hujulikana kama, kichwa kwani ndio hubeba sifa za kategoria ya kirai kile. Nadharia ya Eksibaa huongozwa na "kichwa", ambalo ni neno kuu katika kirai. Kategoria hizi za chini huweza kuwakilishwa na (X). Jerono [15], anasema kuwa, kategoria za juu huweza kuwakilishwa na kategoria za chini katika uchanganuzi kwa kutumia matawi. Kwa mfano;

S5 Mũritwa ũrĩa mwea awîta cukuru -Kiigembe Mwanafunzi yule mzuri ameenda shuleni - Kiswahili 


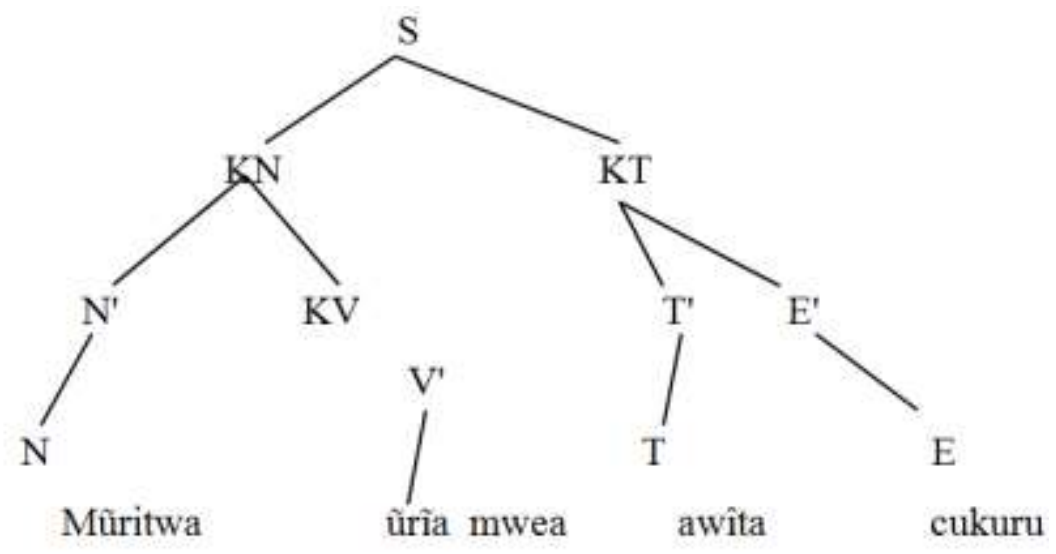

ii) Vipashio katika kirai hupangwa katika ngazi tofauti

Hadhi ya vipashio inapaswa kuonyeshwa vizuri na kwa uwazi zaidi. Ubainishaji wa hadhi ya vipengele vya kileksia ndio huonyesha ni vipashio vipi vinamiliki vipi katika sentensi au kirai. Aidha, ubainishaji huu ndio hudhihirisha vipashio vilivyo katika ngazi sawa au kuwa na uhusiano wa udada. Kichwa cha kirai huwakilishwa kwa X. Hii ndiyo ngazi ya chini kabisa na huwa haina baa yoyote. Ngazi ya pili huwa na baa moja (X') ilhali ile ya tatu huwa na baa mbili (X') na kuendelea hivyo kutegemea muundo wa kirai. Kwa mujibu wa Radford [10], virai huweza kujitokeza katika viwango vitatu, navyo ni kama vifuatavyo;

a)Kategoria ya kiwango cha neno

$\mathrm{N}$ - nomino

T- kitenzi

V- kivumishi

H- kihusishi

E- kielezi

I - kihisishi

b)Kategoria ya kiwango cha kati kama vile;

$\mathrm{N}$ - nomino baa moja

$\mathrm{V}$ - kivumishi baa moja

H- kihusishi baa moja

E- kielezi baa moja

T- kitenzi baa moja

c)Kategoria ya kiwango cha kirai kwa mfano;

RN- kirai nomino

RT- kirai tenzi

KV- kirai kivumishi

KH- kirai kihusishi

KE- kirai kielezi

KI- kirai kihisishi

Radford [10], anabaini kuwa kategoria za kati huwa kubwa kuliko kategoria kileksia lakini ndogo kuliko kategoria kamili (Kirai kikuu). Kuna viambajengo vilivyo vikubwa kuliko nomino lakini vidogo kuliko kirai nomino na jinsi ambavyo kuna virai vikubwa kuliko nomino lakini vidogo kuliko Kirai nomino, ndivyo ilivyo kwa mfano;

i. Virai elezi vilivyo vikubwa kuliko vielezi lakini vidogo kuliko virai vielezi kamili. ii. Virai vivumishi vilivyo vikubwa kuliko vivumishi lakini vidogo kuliko virai vivumishi kamili

iii. Virai vitenzi vilivyo vikubwa kuliko vitenzi lakini vidogo kuliko virai vitenzi kamili.

iv. iv.Virai nomino vilivyo vikubwa kuliko nomino lakini vidogo kuliko virai nomino kamili.

Mifano katika sentensi ya Kî̀gembe;

S6 Johana nî [ RN ûria mûrîmi wa mpempe]. - Johana ni yule mkulima wa mahindi

S7 Mwarîmû nî [KV ûrîa mûkuî mûno ].- Mwalimu ni yule mfupi sana

S8 Mwarîmû [RT ûyû nîakuritana aritwa bake].Mwalimu huyu anafunza wanafunzi wake.

Viambajengo vilivyofungiwa kwenye mabano ni virai vya sentensi ya Kî̀gembe vilivyo na maumbo tofauti. Viambajengo hivi ni virai kamili na ndani yake pana virai vya kati kama ifuatavyo;

S6 Mûrîmî = N (Kichwa)

Mûrîmî wa mpempe $=($ Kirai cha kati $)$

ûrîa mûrîmî wa mpempe $=($ Kirai kamili $)$

S7 mûkuî $=$ V (Kichwa)

Mûkuî mûno $=($ Kirai cha kati $)$

Urîa mûkûî mûno = (Kirai kamili $)$

S8 nîakûritana $=\mathrm{T}($ Kichwa $)$

Nîakûritana aritwa bake $=($ Kirai cha kati $)$

ûyû Nîakûritana aritwa bake $=($ Kirai kamili $)$

Kutokana na mifano hapo juu, ni kweli kuwa, kategoria za kati huwa kubwa kuliko kategoria za kileksia lakini ndogo kuliko kategoria kamili. Aidha, kila kategoria ya kirai kina kategoria leksika. Kirai nomino lazima kiwe na nomino, kirai tenzi lazima kiwe na kitenzi, kirai vumishi lazima kiwe na kivumishi jinsi ambavyo imejitokeza katika mifano ya S6 - S8.

\section{iii) Kipambanuzi}

Kipambanuzi ni kipashio kinachobeba maneno ambayo kimuundo si lazima yawepo katika kirai. Kuhusishwa kwa maneno hayo hukusudia tu kufafanua neno kuu. Aghalabu vivumishi ndivyo hutumiwa kama vipambanuzi katika virai nomino ilhali vielezi ndivyo 
hutumiwa kama vipambanuzi katika virai vitenzi. Kwa mfano;

S6 Johana nî ûria mûrîmi wa mpempe - Kiigembe Johana ni yule mkulima wa mahindi - Kiswahili Kipambanuzi ni kivumishi kionyeshi "yule" ambacho kinafafanua nomino mkulima .

\section{iv) Kijalizo}

Kijalizo ni sehemu ya kirai ambayo huwa imeunganishwa kwa karibu sana na kichwa cha kirai hicho. Kila aina ya kirai huwa na kijalizo chake. Kijalizo huwa na uhusiano wa udada na kichwa cha kirai ;mfano,

S9 Mwiti nî ûmûûme- Kiigembe

Mwiti ni mwerevu - Kiswahili

kijalizo kinatokea kwenye kirai kitenzi na kijalizo hicho ni kivumishi "mwerevu".

\section{v) Kipanuzi}

Kipanuzi ni sehemu ya kirai ambayo hutoa maelezo zaidi kuhusu kirai na huwa hakina uhusiano na kichwa, kipambanuzi au kijalizo. Kwa mfano katika kirai nomino cha sentensi:

S10 Mwarî ûrîa mwikumia aûkoloma - Kî̀gembe Msichana yule mwenye maringo ameketi - Kiswahili

Kipanuzi ni "mwenye maringo." Kirai kinaweza kubeba kipanuzi kimoja,viwili,vitatu au zaidi. mfano; Msichana yule mwenye maringo, ambaye ni mfupi ameketi.

Kuna njia mbalimbali ambazo hutumika katika kuchanganua muundo wa sentensi. Kwa mfano; matawi na mabano. Matumizi ya mabano ya mraba hutenganisha vipashio vyote vilivyomo katika kiambajengo kwa kutumia mabano hayo. Kiambajengo huvidhibiti viambajengo vidogo vinavyounda kirai au sentensi. Mfano ufuatao unaonyesha uchanganuzi wa muundo wa sentensi kwa njia ya mabano:

S11 Mûritwa ûmûraya akwîja - Kîigembe

Mwanafunzi mrefu amefika - Kiswahili

[KN [N mûrîtwa] [V ûmûraya][T akwîja].

Neekemiah [17], anaeleza kuwa; ni vizuri kuchanganua miundo ya kisintaksia kwa kuangazia uchanganuzi wa kategoria ya virai na si kwenye mkabala wa maneno tu. Kutokana na hali hiyo, utafiti huu ulitumia nadharia hii kujaribu kuonyesha miundo mbalimbali ya virai vya sentensi ya Kî̀gembe.

Katika utafiti huu wa miundo ya virai vya sentensi ya Kî̀gembe ilichanganuliwa kwa kutumia matawi ya kisintaksia kwa sababu, matawi huonyesha uhusiano wa moja kwa moja wa vipashio vinavyounda viambajengo vya sentensi kama vile; virai. Kwa kawaida matawi haya huchomoza kutoka kwenye viambajengo. Kila kifundo huwakilisha kiambajengo kilichomo katika muundo wa kirai au sentensi. Kifundo kinachokaa juu mti wa kisintaksia hufahamika kama kifundo cha juu na ndicho kiambajengo kikubwa. Kifundo hiki ndicho hutawalia vifundo vya chini ambavyo hubeba viambajengo vidogo. Katika matawi haya, vifundo vya daraja la chini hupangana kuonyesha kipashio kipi hutangulia kingine. Mfano wa sentensi ya Kî̀gembe;

S12 Mwekûrû ûyû mûkuî endete kûrîma rûkîrî mûnoKiigembe

Mama huyu mfupi anapenda kulima asubuhi sanaKiswahili

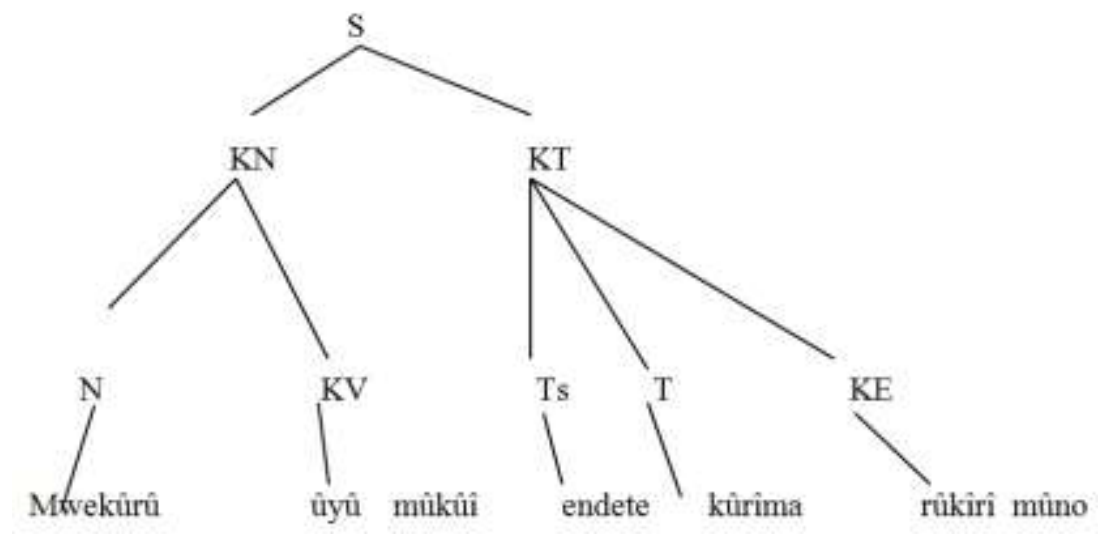

Aidha, nadharia ya Eksibaa huwa na vifundo, vifundo hivi hupangana kisafu na hujulikana kama vifundo dada ambavyo hutawaliwa moja kwa moja na kifundo cha juu [16]. Kwa mfano, ikiwa KN kitakuwa ni kifundo cha juu kitatawalia na $\mathrm{N}$ na $\mathrm{V}$ ambavyo vitakuwa ni vifundo vya chini na pia ni viambajengo vidogo vya kifundo cha juu.

\section{Ukusanyaji wa Data}

Ili kupata data ya makala hii watafiti walitumia chanzo cha data zaidi ya kimoja ili kupata data zinazofaa na za kutosha kutoa matokeo bora. Njia kuu ilikuwa watafiti kuzalisha zile sentensi wenyewe kwa kuwa wazawa wa lahaja na pia kukusanya data nyanjani ili kuondoa shaka la kuwa na upendeleo. Jumla ya data ya sentensi zilizokusanywa ni sentensi 50. watafiti walitunga data ya sentensi ya Kî̀gembe na pia 
kukusanya nyanjani na kuichanganua kwa misingi ya Nadharia ya Eksibaa. Aidha, jumla ya sentensi 50 zilitumika kuchanganua muundo wa sentensi ya Kî̀gembe. Sentensi hizi zilitosha kushughulikia aina tatu za sentensi za Kî̀gembe zilizoshughulikiwa katika makala hii ambazo ni; sentensi sahili, ambatano na changamano. Kulingana na Chomsky [9], akijibu swali ni kivipi mzawa wa lahaja anayezalisha sentensi ataepukana na upendeleo, anasema kuwa, kuna aina mbili za umilisi yaani, umilisi wa kisanyansi na umilisi kisarufi. Aidha, anasema umilisi wa mzawa wa lugha yeyote ile hudhihirika kwa kuwa na ufahamu wa fonolojia, sintaksia na semantiki ya lugha hiyo. Ni kutokana na ukweli huu anasema kwamba, mzawa wa lugha ana uwezo wa kuunda sentensi kamilifu kutokana na kuelewa sauti za lugha yake na zilizo na maana iliyokusudiwa bila upendeleo au utata wowote ule kwani anaelewa sentensi zilizo na kasoro. Kwa hivyo, suala hili lina maana kuwa, mzawa wa lugha anaweza kuzalisha sentensi bila upendeleo. Hata hivyo, watafiti walitafiti nyanjani ili kuondoa shaka la upendeleo na kufanikisha kupata jumla ya sentensi 50 zilizochanganuliwa.

\section{Uchanganuzi wa Data}

Miundo ya virai hujikita katika neno kuu au kichwa cha kirai. Kulingana na Leffel na Bouchard [14], neno kuu katika kategoria za virai ndio husimama kama neno kuu ambalo ni; kitenzi, nomino, kielezi, kivumishi au kihusishi. kwa hivyo, aina za maneno katika sentensi ya Kî̀gembe ndizo hutawala virai kulingana na mahusiano ya maneno katika sentensi. Virai huainishwa na aina tano za maneno yaani; virai nomino, virai tenzi, virai elezi, virai vihusishi na virai vivumishi. Aina za maneno kama vile viwakilishi huchukuliwa kama virai nomino kutokana na uhusiano kati ya nomino na viwakilishi vya nomino. Aidha, vihisishi huchukuliwa kama virai elezi, huku viunganishi vikichukuliwa kama virai vihusishi kulingana na uhusiano wake.

\section{Miundo ya Virai Nomino}

Kwa mujibu wa Massamba na wenzake [4], kirai nomino ni kirai ambacho muundo wake hutegemea nomino au mahusiano ya nomino na maneno mengineyo katika sentensi. Kama ilivyoelezwa hapo awali uhusiano wa kiwakilishi na nomino huwa ni wa karibu sana. Kwa hivyo, kiwakilishi kilichukuliwa kama nomino mahali ambapo kinatokea katika sentensi ya Kî̀gembe.

Sentensi ya Kî̂gembe ina miundo ya kirai nomino ifuatayo;

\section{(a) Kirai nomino cha nomino moja pekee.}

Aina hii ya kirai nomino huundwa na neno moja tu ambalo ni nomino au kiwakilishi cha lugha ya Kî̀gembe. Kwa mfano;

S13 Mûritwa awîta cukuru (Kî̀gembe)
Mwanafunzi ameenda shule. (Kiswahili)

S14 Ntîî nîakûrimira mwarocia (Kî̀gembe)

Mama anamlimia dadangu (Kiswahili)

S15 Nîwe ûkareta mûîkania (Kî̀gembe)

Wewe utaleta bwana harusi (Kiswahili)

Muundo wa kirai nomino cha nomino moja pekee ya Kî̀gembe unaonekana kwa kielelezo cha mchoro kama ifuatavyo;

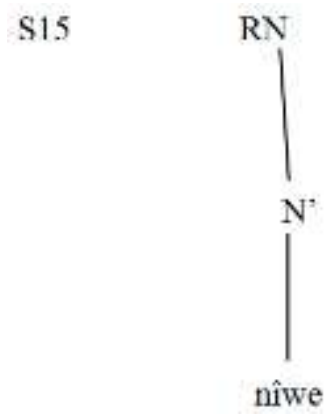

Katika sentensi za S13- S15 virai nomino vya muundo wa neno moja ni mûritwa, ntîî na nîwe ni virai nomino ambavyo vimesimama kama neno kuu la kirai nomino kwani vimejengwa na nomino moja au kiwakilishi chake kama mfano wa S15. Kama ilivyoelezwa hapo awali ni kuwa kiwakilishi huchukuliwa kuwa nomino kwa sababu ya kuwepo na uhusiano wa karibu kwani kiwakilishi huweza kusimamia nomino katika sentensi ya Kî̀gembe.

Kulingana na Nadharia ya Eksibaa, Radford [12], anasema kuwa, kila mahali ambapo kirai kinatokea lazima kuwe na kategoria ya leksia. Kategoria hizi za maneno ndizo hubeba sifa za kirai. Hii ni kumaanisha kuwa, kila kunapotea kirai nomino lazima kuwe na nomino jinsi ambavyo imewasilishwa na S14. Nadharia ya Eksibaa huongozwa na kichwa ambalo ni neno kuu la kirai. Katika Nadharia ya Eksibaa kibainishi huweza kupanua $\mathrm{N}$ ' hadi $\mathrm{N}$ ' ila wakati ambapo kirai nomino huwa na muundo wa nomino pekee huweza kujipanua hadi kiwango cha $\mathrm{N}$ ',

\section{(b) Kirai nomino cha nomino na kishazi tegemezi}

Sentensi ya Kị̂gembe huwa na kirai nomino ambacho kinaundwa na nomino na kishazi tegemezi. Mifano ifuatayo imethibitisha.

S16 Mwiki ûrîa ûraikîrue îoro nî ûmûnthongi (Kî̀gembe)

Bibi arusi aliyeolewa jana ni mrembo (Kiswahili) S17 Miraa îria îraûrîrwe îoro nî yeetû (Kî̀gembe) Miraa iliyonunuliwa jana ni yetu (Kiswahili) S18 Mûate yûrîa yûraûrîrwe yûkûthûka (Kî̀gembe) Mkate ulionunuliwa umeharibika (Kiswahili)

Muundo wa kirai nomino cha nomino na kishazi tegemezi cha Kî̂gembe kinajitokeza kwa namna ifuatayo; 
S16

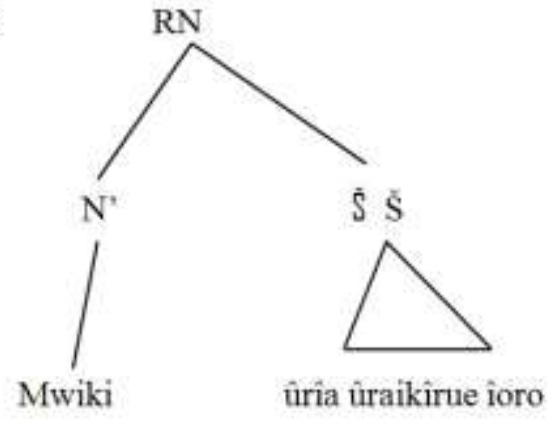

Kwa mujibu wa Nadharia ya Eksibaa kipanuzi ni sehemu ya kirai ambayo hutoa maelezo zaidi kuhusu kirai. Kirai kinaweza kubeba kipanuzi kimoja, viwili au zaidi. Katika mifano ya S16 - S18 vishazi tegemezi vimetumika kama vipanuzi. Kipanuzi si kategoria ya lazima katika kirai kwani vishazi tegemezi katika mifano ambayo imetumika inaweza kutolewa na kirai kiweze kujipanua. Maneno yaliyoandikwa kwa herufi za mlazo ni muundo wa kirai nomino cha Kî̀gembe ulio na kirai nomino na kishazi tegemezi kwa mfano; Mwiki ûrîa ûraikîrue îoro, miraa îrîa îraûrîrwe îoro na mûate yûrîa yûraûrîrwe. Vishazi tegemezi vinapotumika pamoja na nomino hufanya kazi ya kuvumisha nomino ile imetumika. Kwa mifano; ûrîa ûraikîrue îoro îrîa

s19 îraûrîrwe îoro na yûrîa yûraûrîrwe ni vishazi tegemezi vinavyovumisha nomino zilizo katika S16 - S18.

(c) Kirai nomino cha nomino na kivumishi

Kivumishi hiki huwa kinaelezea nomino ambayo ni kichwa cha kirai moja kwa moja. Kivumishi katika muundo huu kinaweza kuwa kimoja au zaidi. Nadharia ya Eksibaa hueleza dhana ya kipambanuzi kama kipashio kinachobeba maneno ambayo kimuundo si lazima yawepo katika kirai. Aidha, Nadharia ya Eksibaa inatilia mkazo kuwa, kuhusishwa kwa kivumishi kimoja au zaidi hukusudia tu kufafanua neno kuu (kichwa). Aghalabu vivumishi ndivyo hutumiwa kama vipambanuzi katika virai nomino ilhali vielezi hutumika kama vipambanuzi katika virai vitenzi. Kwa mfano;

S19 Mûate yûû nî yûmwea (Kî̀gembe)

Mkate huu ni mzuri (Kiswahili)

S20 Mwekûrû ûyû ûmûraya akûra munda (Kî̀gembe) Mama huyu mrefu amenunua shamba (Kiswahili)

S21 Maûta yakwa yakûthira (Kî̀gembe)

Mafuta yangu yameisha (Kiswahili)

Muundo huu unaweza kuwakilishwa katika mchoro kama ifuatavyo;

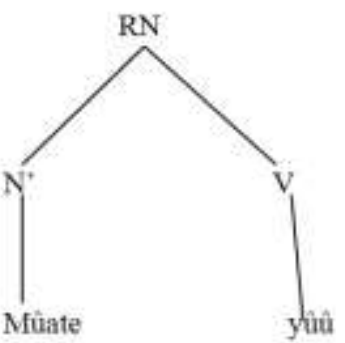

20

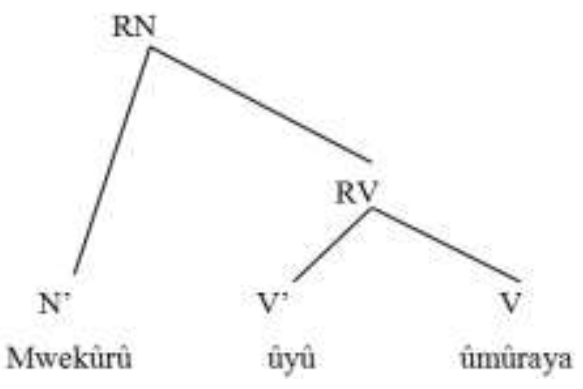

Kulingana na mifano S19 - S21 ni mifano inayoonyesha muundo wa kirai nomino (kilicho katika herufi za mlazo) ambacho kinaundwa na nomino na kivumishi. Vivumishi kulingana na Nadharia ya Eksibaa vinafanya kazi ya kupanua nomino. Hata hivyo, kirai nomino cha Kî̀gembe kinaweza kuchukua muundo wa nomino na vivumishi viwili au zaidi kwa mfano, S21. (d) Kirai nomino cha kiwakilishi pekee

Hiki ni kirai nomino ambacho muundo wake ni wa kiwakilishi pekee. Hapo awali ilielezwa kuwa kiwakilishi huchukuliwa kama nomino kwa sababu ya uhusiano wake na nomino. Kiwakilishi hutumika kuwakilisha nomino mahali ambapo nomino haijaweza kutumika. Mifano ya kirai hiki ni kama ifuatayo, S22 Wee nîakûthambîa mwaana (Kî̀gembe) Yeye anaosha mtoto (Kiswahili) S23 Kîi kîkûrîrwa mûrîtwa (Kî̀gembe) Hiki kimenunuliwa mwanafunzi (Kiswahili) 
Muundo wa kirai nomino cha nomino na kivumishi unaweza kuwakilishwa kwa kielelezo kifuatacho;

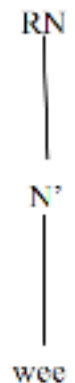

Mifano hii iliyoandikwa kwa herufi za mlazo zinaonyesha mifano ya virai nomino vya Kị̂gembe ambavyo vinaundwa na kiwakilishi pekee. Nadharia ya Eksibaa huongozwa na kichwa ambalo ni neno kuu katika kirai. Katika utafiti huu uliweza kuangazia aina tano za virai yaani virai nomino, virai elezi, virai tenzi, virai vivumishi na virai husishi. Aina za maneno huwa nane lakini virai huwa aina tano hii ni kwa sababu kategoria za maneno ambazo huwa na uhusiano kama vile, vihisishi na viunganishi huchukuliwa kama virai husishi huku viwakilishi vikichukuliwa kama virai nomino kila mahali ambapo kiwakilishi kinapotokea. Maneno yaliyoandikwa kwa herufi za mlazo katika mifano ya S27-S28 ni viwakilishi ambavyo ni virai nomino.

\section{(e) Kirai nomino chenye muundo wa kiwakilishi na kivumishi}

Kirai nomino cha Kîngembe huundwa na kiwakilishi na kivumishi. Mifano ifuatayo inaelezea zaidi.

S24 Yûû mûraya yûatemwa (Kị̂gembe)

Huu mrefu utakatwa (Kiswahili)

S25 Yaa maîngî nî ya?(Kîîgembe)

Haya mengi ni ya nani? (Kiswahili)

S26 Kî̀ kînene kîatûka nîkîetû (Kî̀gembe)

Hiki kikubwa kimevunjika ni chetu (Kiswahili)

Kirai nomino cha Kî̀gembe chenye muundo wa kiwakilishi na kivumishi, kimewakilishwa na; yûû mûraya, yaa maîngî na kîi kînene. Muundo wa kirai hiki unaonekana
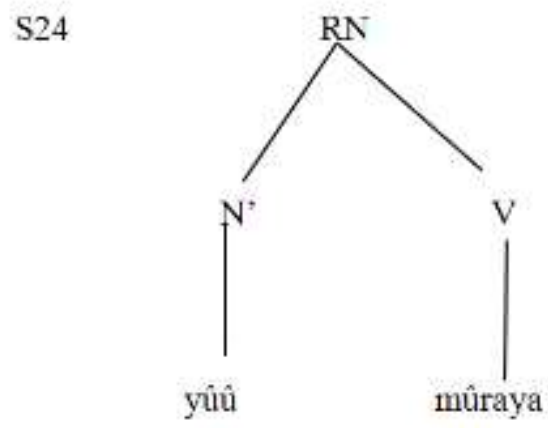

Katika mifano S24- S26, vivumishi ambavyo vimetumika vinafanya kazi ya kupambanua kirai nomino ambacho kimewakilishwa na viwakilishi katika sentensi hizo.

\section{(f) Kirai nomino chenye muundo wa kitenzijina}

Nadharia ya Eksibaa huongozwa na dhana ya kichwa (neno kuu) katika kirai na uhusiano wake na vipengele vingine ambavyo vimetumika kuunda kirai. Nadharia ya Eksibaa husaidia kuchanganua sentensi kwa kutekeleza jukumu kama vile; kubainisha muundo wa kirai. Katika muundo huu wa kirai nomino, Nadharia hii ilitumika kuonyesha muundo wa kirai hiki ambacho ni kirai ambacho kiliundwa na nomino ya kitenzijina pekee. Sentensi ya Kî̀gembe huwa na muundo wa kirai nomino ambacho kina muundo wa kitenzijina. Kwa mfano:

S27 Kûtheka kûkatûwîrîa mûno (Kî̀gembe)

Kucheka kutatufurahisha sana (Kiswahili)

S28 Kûîna nîkûkwea (Kî̀gembe)

Kuimba ni kuzuri (Kiswahili)

Muundo wa kirai nomino cha kitenzijina kina muundo ufuatao katika Kî̀gembe, S27

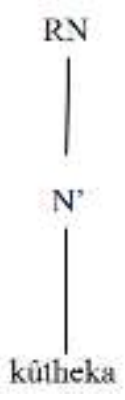

Mifano iliyoandikwa kwa herufi za mlazo ni mifano ya vitenzi jina vya Kî̀gembe ambavyo vinatumika kuundia kirai nomino hiki. Hivi ni kama vile; kûtheka na kûîna.

\section{(g) Kirai nomino cha muundo wa kitenzi jina na kivumishi}

Hiki ni aina ya kirai cha Kî̀gembe ambacho huundwa kwa kutumia kitenzijina na kivumishi. Mifano ifuatayo inadhibitisha hilo.

S29 Kûîna kwake nî kûtûwîria (Kî̀gembe)

Kuimba kwake kunafurahisha (Kiswahili)

S30 Kûcetha kûû tî kûkwea (Kî̀gembe)

Kucheza huku si kuzuri (Kiswahili)

S31 Kûthoma kwake nî kwakûrîarania (Kî̀gembe)

Kusoma kwake ni kwa kushangaza (Kiswahili)

Katika mifano hii iliyotolewa yenye muundo wa kirai nomino cha Kî̂gembe chenye muundo wa kitenzijina na kivumishi unaweza kujitokeza ifuatavyo; 
$\mathrm{S} 30$

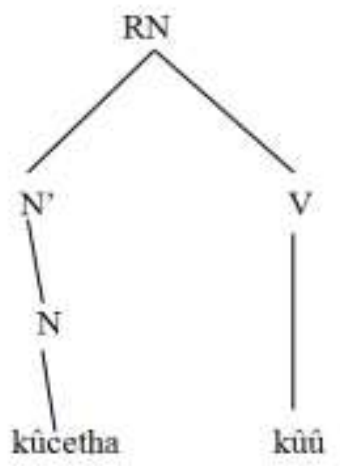

Nadharia ya Eksibaa huzingatia upangaji wa vipashio mbalimbali vya kileksia katika viwango tofauti katika sentensi. Ni upangaji unaobaini ni kipashio kipi kina uhusiano wa ngazi moja na ni kipi kinamiliki kingine. Radford [12], alitumia istilahi kama vile; dada (viambajengo vilivyo katika ngazi moja) kwa mfano; $N^{\prime}$ na $V^{\prime}$ viko katika ngazi moja, na binti ya (viambajengo vinavyomilikiwa na vingine) kwa mfano tulioutoa S35 N' na V' ni binti wa N' ambacho ndicho kirai kamili. Katika muundo huu wa kirai cha Kî̀gembe chenye muundo wa kitenzijina na kivumishi vimeweza kuwakilishwa na; Kûîna kwake, kûcetha kûû na Kûthoma kwake.

\section{(h) Kirai nomino chenye nomino mbili}

Kirai cha Kî̀gembe kinaweza kujitokeza na muundo wa kirai nomino chenye kichwa ambacho ni cha nomino mbili. Mifano ifuatayo inaweza kuthibitisha.

S32 Mwekûrû o mûthoi nî ûyû (Kî̀gembe)

Mke wa msasi ni huyu (Kiswahili)

S33 Mûwati o mwana awîta (Kî̀gembe)

Mlezi wa mtoto ameenda (Kiswahili)

S34 Mwarîmû o twaana nîakûrîtana (Kî̀gembe)

Mwalimu wa watoto anawafunza (Kiswahili)

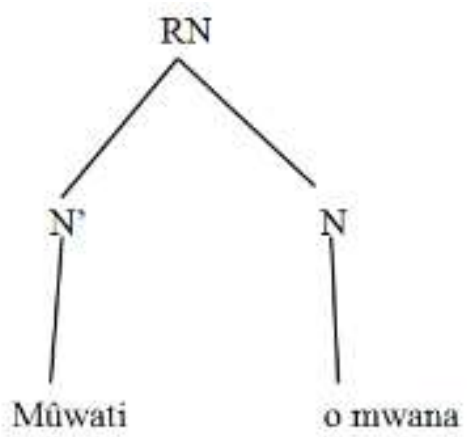

Mifano S32- S34 iliyoandikwa kwa herufi za mlazo ni mifano ya virai vya nomino vya Kî̂gembe vyenye muundo wa nomino mbili ambavyo ni kama vile; Mwekûrû o mûthoi, mûwati o mwana na mwarîmû o twana.

\section{Miundo ya Virai Vivumishi}

Virai vivumishi ni aina ya virai ambavyo muundo wake huundwa kwa kivumishi. Ni fungu la maneno lenye kivumishi ambacho ndicho kichwa au neno kuu la kirai hicho. Kirai vumishi hufanya kazi ya kutoa maelezo zaidi juu ya nomino au kiwakilishi chake. Aghalabu kirai cha Kî̀gembe hujitokeza katika kirai cha nomino na kikundi tenzi hasa baada ya kikundi tenzi chenye muundo wa kitenzi kishirikishi. Kirai kivumishi cha Kî̀gembe kinaweza kujitokeza kwa miundo ifuatavyo;

(a) Kirai kivumishi chenye kivumishi pekee. S35 Mûrîtwa ûmuraya aûkîruka kîerîo. (Kî̀gembe) Mwanafunzi mrefu amepita mtihani (Kiswahili) Muundo wa kivumishi unaweza kujitokeza kama ifuatavyo;

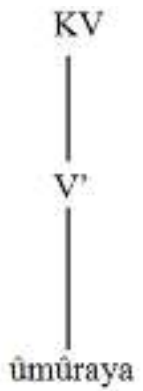

Neno lililokoleshwa rangi ni kirai kivumishi chenye muundo wa kivumishi pekee.

(b) Muundo wa kirai kivumishi chenye muundo wa zaidi ya kivumishi kimoja

S36 Mûrîtwa ûmuraya ûmûthongi aûkîruka kîerîo. (Kîigembe)

Mwanafunzi mrefu mrembo amepita mtihani (Kiswahili)

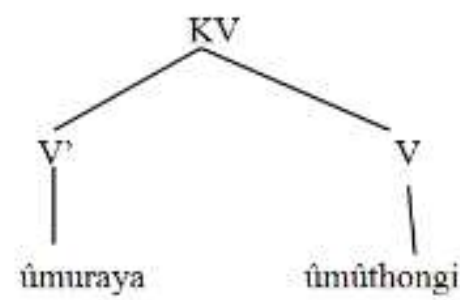

Kulingana na Cowper [10] muundo wa virai huchukuliwa kuwa na viwango vitatu vya uchomozi ambavyo ni; kiwango cha uchomozi wa neno, kiwango cha uchomozi wa kati na uchomozi wa upeo. Kiwango cha uchomozi upeo huwakilishwa na herufi ya neno kuu katika kirai pamoja na baa mbili juu yake ( $\left.X^{\prime \prime}\right)$. Kwa upande mwingine uchomozi wa kati huwakilishwa na herufi ya neno kuu katika kirai pamoja na baa moja (X'). Uchomozi wa neno huwakilishwa na alama (X). Uchomozi ambao unajitokeza katika mifano hii ni wa (X) ambao umethibitishwa kwa mchoro wa S40. 


\section{(c) Kirai kivumishi chenye kivumishi na kielezi}

Kirai kivumishi cha Kî̀gembe kinaweza kuchukua muundo wa kivumishi na kielezi kama inavyojitokeza katika mifano ifuatayo;

S37 Mwana ûmûkuî mûno nîakûrîra (Kî̀gembe)

Mtoto mfupi sana analia (Kiswahili)

S38 Mwarîmû ûmûme mûno nîakûandîka (Kị̂̂gembe)

Mwalimu mwerevu sana anaandika (Kiswahili)

Muundo wa kirai kivumishi cha Kî̀gembe chenye muundo wa kivumishi na kielezi unaweza kujitokeza ifuatavyo,

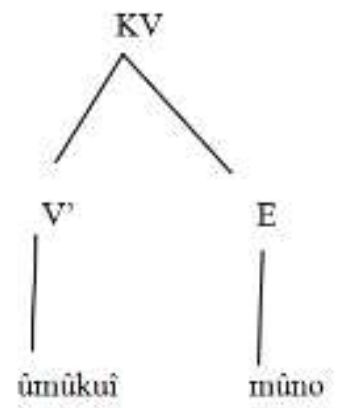

Vielezi katika virai vivumishi hivi vya S41S42 vilitumika kama kipambanuzi ambacho kinakamilisha kirai kivumishi.

\section{(d) Kirai kivumishi chenye kivumishi na nomino}

Muundo wa kirai kivumishi unajitokeza kuwa na kivumishi na nomino kulingana na mifano hii inayothibitisha hilo.

S39 Mwekûrû wa îtonga akwîja (Kî̀gembe)

Mke wa tajiri amekuja (Kiswahili)

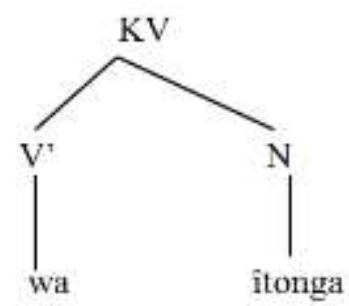

Kirai kivumishi cha Kî̀gembe chenye muundo wa kivumishi na nomino hujitokeza aghalabu katika vivumishi vya a-unganifu. Aidha, kulingana na Nadharia ya Eksibaa kila kirai huongozwa na neno kuu. Neno kuu katika mifano ya S43 ni kivumishi na hii ndio sababu, utafiti huu ulibainisha kuwa mfano huu ni wa kirai kivumishi.

\section{Miundo ya Virai Elezi}

Hivi ni virai vya sentensi ya Kî̂gembe ambavyo hutumika kutolea maelezo zaidi juu ya kivumishi, kitenzi au kielezi kingine. Kwa mfano;

(a) Kirai kielezi chenye kielezi pekee

S40 Ntîî awîta mundene (Kî̀gembe)
Mama ameenda shambani (Kiswahili)

Muundo huu unaweza kujitokeza ifuatavyo;<smiles>[14CH3][14CH3]</smiles>

Kirai kielezi ambacho ni cha kielezi pekee kimetumika kujipanua kwani kulingana na Nadharia ya Eksibaa kirai cha neno huweza kujipanua kutoka E- E' hadi E",

(b) Kirai kielezi chenye kielezi zaidi ya kimoja

S41 Mwarîmû akûritana bwea mûno (Kị̂gembe) Mwalimu amefunza vizuri sana (Kiswahili) Muundo wa kirai kielezi inaweza kujitokeza ifuatavyo;

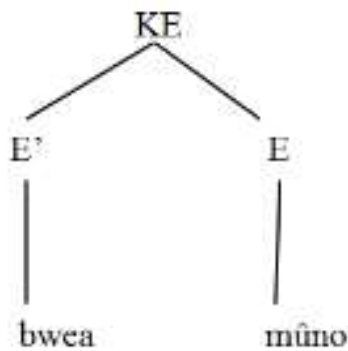

Kulingana na Nadharia ya Eksibaa, kijalizo ni sehemu ya kirai ambayo huwa imeunganishwa kwa karibu sana na kichwa cha kirai hicho. Kila aina ya kirai huwa na kijalizo chake. Kijalizo huwa na uhusiano wa udada na kichwa cha kirai. Katika mfano S41 neno mûno ni kijalizo cha kirai kielezi kwani linaelezea zaidi kuhusu bwea. Aidha, linaonyesha uhusiano wa udada katika tungo hilo kwani E kinapambanua E' hadi E'.

\section{Miundo ya Virai vihusishi}

Kirai husishi, kama ilivyo aina nyingine za virai huwakilishwa na neno kuu yaani kichwa cha kirai ambacho ni kihusishi. Aghalabu kirai husishi hutokea sehemu ya kikundi nomino na kikundi tenzi iwapo kitatokea upande wa kikundi nomino basi hufanya kazi kama kielezi. Hata hivyo, kitokeapo upande wa kikundi nomino hufanya kazi ya kuvumisha. Miundo ya virai vihusishi inaweza kujitokeza ifuatavyo;

(a) Kirai husishi chenye kihusishi na kielezi S42 Mpempe cia mundene ikûma (Kî̂gembe) Mahindi ya shambani yamekauka (Kiswahili) S43 Aini ba kanicene bakwiya (Kị̂̂gembe) Waimbaji wa kanisani wamefika (Kiswahili)

Muundo huu wa kirai husishi unaweza kuwasilishwa kwa namna ifuatayo; 


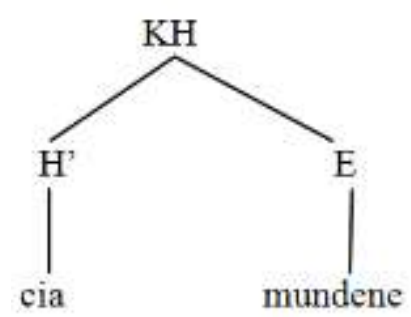

Muundo huu wa kirai husishi maneno yaliyoandikwa kwa herufi za mlazo ni virai vihusishi. Ilibainika kuwa virai husishi vya Kî̀gembe vinaweza kutumika kama vielezi vinapotumika pamoja na kielezi.

(b) Kirai husishi chenye muundo wa kihusishi na nomino

S44 Biakûrîa vya ngûûûu bîkûya (Kị̂gembe)

Vyakula vya nyanya vimeiva (Kiswahili)

S45 Kîratû kîa mûranû nî kîthongî (Kî̀gembe)

Kiatu cha harusi ni kizuri (Kiswahili)

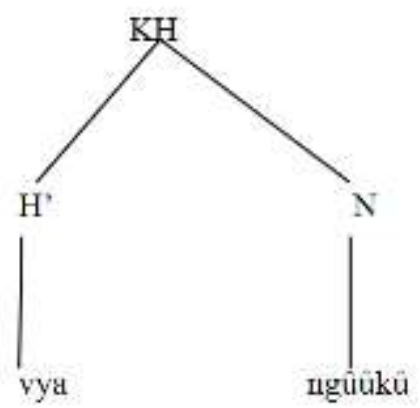

Virai husishi ambavyo vimekoleshwa rangi ni mfano wa virai vya Kî̀gembe vinavyochukua muundo wa kihusishi na nomino. Katika nadharia ya Eksibaa kipambanuzi ni kipashio kinachobeba maneno ambayo hukusudia tu kufafanua neno kuu. Katika mifano ya S44-S45, maneno kama vile, ngûûkû na mûranû ni mifano ya vipambanuzi katika virai hivi vihusishi kwani vinatumika kuelezea vihusishi.

\section{Miundo ya Virai Vitenzi}

Kirai Kitenzi ni kirai ambacho kimeundwa katika mahusiano ya kitenzi na mafungu ya maneno [4]. Kama kawaida ya virai neno kuu katika kirai kitenzi huwa ni kitenzi. Kirai kitenzi ni mojawapo wa kipashio cha lazima katika sentensi ya Kî̂gembe. Miundo ya kirai kitenzi ni kama ifuatayo;

(a) Kirai kitenzi chenye kitenzi halisi pekee.

S46 Akûwa (Kî̀ngembe)

Ameanguka (Kiswahili)

S47 Nîakûthoma (Kî̀gembe)

Anasoma (Kiswahili)
RT

nîakûthoma

Muundo huu ni wa kirai kitenzi kimoja ambacho kinaweza kujisimamia kama sentensi ya Kî̀gembe. Kichwa cha kirai ambacho ni neno kuu la kirai huweza kuwa neno moja ambalo hubeba sifa za kirai kikuu. Katika mifano hii inathibitisha muundo wa kirai ambacho kimeundwa na neno moja ambalo ni kitenzi cha pekee.

\section{(b) Kirai kitenzi chenye muundo wa kitenzi na} kielezi

S48 Mwarîmû akûrîtana mûno (Kî̀gembe)

Mwalimu amefunza sana (Kiswahili)

S49 Munda niyûkûrîmîka bwea (Kî̀ngembe) Shamba linalimika vizuri (Kiswahili)

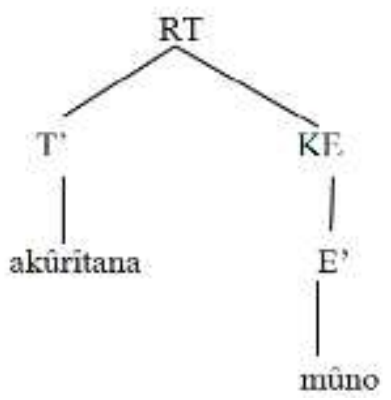

Virai vilivyoandikwa kwa herufi za mlazo ni mfano wa virai vitenzi vyenye muundo wa kitenzi na kielezi. Aidha, katika kielelezo cha matawi S49 neno mûno limetumika kama kijalizo ambacho ni dada ya kitenzi na hupanua kitenzi T' na T' hujinua hadi T'”

(c) Kirai kitenzi chenye muundo wa vitenzi viwili S50 Ndamûtîrîmîre akinaa (Kî̂̀embe)

Nilimkuta akiimba (Kiswahili)

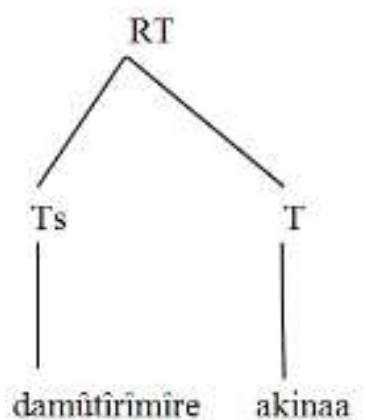


Radford [18] anadai kuwa vitenzi visaidizi ni vitenzi vinavyohitaji vitenzi vikuu hili kukamilishwa. Kitenzi kisaidizi hujalizwa na kitenzi kikuu. Aidha, vitenzi halisi huchukua aina tofauti za kategoria za maneno kuzijaliza.

Muundo wa kirai kitenzi chenye vitenzi viwili aghalabu hujitokeza hasa ambapo sentensi ya Kî̀gembe imewakilishwa na kitenzi kisaidizi na kitenzi kikuu. Katika Nadharia ya Eksibaa kitenzi kikuu kinapoandamana na kitenzi kisaidizi, kinafanya kazi ya kujaliza kitenzi kisaidizi. Kijalizo hutumika kutolea maelezo kuhusu neno kuu ambalo ni kichwa cha kirai.

\section{HitiMisho}

Makala hii imejadili muundo wa kirai cha Kiigembe. Ndharia ya X- baa iliongoza utafiti huu. Ilibainika kuwa Kiigembe kina aina tano ya virai mathalan: kirai nomino, kirai tenzi, kirai kivumishi, kirai elezi na kirai kihusishi. Aidha, uelewa wa muundo huu unafanikisha mawasiliano na kuchangia umilisi wa lugha hii kwa wasemaji asilia. Data iliyozuliwa inaweza kuchangia uelewa wa lugha zingine za kibantu zenye muundo wa aina hii.

\section{MAREJELeO}

1. Chomsky, N. (1970). Aspect of the Theory of Syntax. Cambridge: MIT Press.

2. Mkude, J. J. D. (1983). Uchanganuzi wa Sentensi za Kiswahili: Dar es salaam: TUKI

3. Besha, R. M. (1994). Utangulizi wa Lugha na Isimu. Dares salaam. Macmillan Aidan Ltd.

4. Massamba, na wenzake (1999). Sarufi Miundo ya Kiswahili Sanifu (SAMIKISA). Sekondari na Vyuo, Dares-salaam: Taasisi ya Uchunguzi wa Kiswahili.

5. Khamisi, A. A. (2011). Uchambuzi wa Kiswahili Fasaha: Sarufi na Lugha, Zanzibar: Kikuu cha Elimu Chukwani.
6. Buliba, A., Njogu, K., \& Mwihaki, A. (2006). Isimujamii kwa wanafunzi wa kiswahili. Jomo Kenyatta Foundation.

7. Rubanza, Y. I. (2003). Sarufi: Mtazamo wa Kimuundo, Dares salaam.Chuo Kikuu cha Huria cha Tanzania.

8. Radford, A. (1981). Transformaional Syntax. Cambridge: Cambridge University Press.

9. Chomsky, N. (1986). Knowledge of Langauge, Its Nature, Origin and Age. New York: Praeger.

10. Cowper, E. (1992). A Concise Introduction to Syntactic Theory: The Government-Bindin Aproach: Chicago, The University of Chicago Press.

11. Newmeyer, F. J. (1986). Linguistic Theory in America 7th Edition Orlando:Academic Press Inc. Chicago University Press.

12. Radford, A. (1988). Small children's small clauses. Transactions of the philological society, 86(1), 1-43.

13. Buberwa, A. (2011). Muundo wa Majina ya Mahali katika Kiswahili: Uchunguzi Kifani wa Majina ya Vituo vya Daladala Jijini Dar es Salaam. Kioo cha Lugha, 9(1), 1-14.

14. Leffel, K. na Bouchard, D. (1991). Views on Phrase Structure Kluwer Academic Publishers, Dordrecht, The Nertherlands.

15. Jerono, P. (2003). Kishazi Huru Arifu cha Kiswahili: Mtazamo wa x-baa.(Tasnifu ya Uzamili haiyachapishwa) Chuo Kikuu Cha Nairobi.

16. Fromkin, V. A. (2000). Brain, language, and linguistics. Brain and language, 71(1), 72-74.

17. John, M. F., \& Kobia, J. (2020). Muundo wa Virai vya Sentensi ya Kî̂gembe katika Kuafikia Maendeleo Endelevu kwa Waigembe. Research Journal in African Languages, 1(1), 23-50.

18. Radford, A. (2004). English syntax: An introduction. Cambridge University Press.

Cite This Article: Mutwiri Fred John M. A \& Allan Mugambi (2021). Muundo WA Virai Vya Sentensi Ya Kî̂gembe. East African Scholars J Edu Humanit Lit, 4(3), 129-141. 